gänzt werden, so resümiert Greven, „dominiert am Ende doch die legitimierte Amtsgewalt oder Mehrheitsentscheidung“" (S. 338).

Insgesamt handelt es sich bei dem vorliegenden Tagungsband um eine interessante und lesenswerte Zusammenstellung von Beiträgen, die sich auf unterschiedliche Weise dem Phänomen politische Reformen nähern. Im Kontext der Reformdiskussion liegt dem Titel des Buches - Staat und Gesellschaft - implizit eine gängige Unterscheidung aus dem Bereich der politischen Steuerungswissenschaft zugrunde: Von Bedeutung ist damit nicht nur die Steuerungsfähigkeit (Staat), sondern gleichzeitig die Steuerbarkeit unterschiedlicher Teilsektoren (Gesellschaft), um Veränderungen wirkungsvoll ins Werk setzen zu können. Trotz eines positiv ausfallenden Gesamturteils verbleiben einige einschränkende Anmerkungen. So hätte es das Bild abgerundet, wenn die Rolle der Parteien und des Bundestages als Promotoren von Innovationen und Reformen stärker beleuchtet worden wäre. Bei der Auswahl der einzelnen Politikfelder hätte man sich gewünscht, dass insbesondere jene Bereiche thematisiert worden wären, die seit vielen Jahren kontrovers diskutiert werden und in der Öffentlichkeit symptomatisch für den deutschen „Reformstau“ stehen (vor allem das Steuersystem und der Gesundheitssektor). Gerade im Abschnitt Reform ohne Staat hätte man gern erfahren, ob erfolgreiche Veränderungen in Politik und Gesellschaft nicht auch im Gewand nicht-intendierter Nebenfolgen staatlichen Handelns daherkommen können. Am Ende sei das Buch insbesondere all jenen Beobachtern und Kommentatoren als aufschlussreiche Lektüre empfohlen, die zwar gescheiterte Reformen zur Kenntnis nehmen, die systemeigenen Erfolgsbedingungen politischer Reformanstrengungen in der Bundesrepublik jedoch kaum berücksichtigen.

Helge F. Jani

\title{
Edierte Protokolle der Bundestagsfraktion der Grünen
}

\section{Boyer, Josef und Helge Heidemeyer (Hrsg.): Die Grünen im Bundestag. Sitzungsprotokolle} 1983-1987, Droste Verlag, Düsseldorf 2008, 2 Halbbände, 1137 Seiten, € 140,-.

Die Grünen sind historisch geworden. Vorbei sind die Zeiten, da man ausrechnen musste, mit welcher Wahrscheinlichkeit die Partei über die Fünf-Prozent-Hürde kommen werde. Von den frühesten Grünen Listen an gerechnet, sind die Grünen nun schon mehr als vierzig Jahre präsent, gerade haben sie ihr 25jähriges Jubiläum als Bundestagsfraktion gefeiert. Im Fünfparteiensystem der Bundesrepublik erwachsen ihnen neue Chancen. Sie sind als eine der Grundströmungen des Parteiensystems nicht mehr wegzudenken.

Immer noch klagt das Feuilleton, sie hätten sich zu sehr „angepasst“. Jetzt gibt es Gelegenheit, die wilde Geschichte der Grünen noch einmal in ihren Anfängen nachzulesen. Die Kommission für Geschichte des Parlamentarismus und der politischen Parteien hat verdienstvollerweise die Sitzungsprotokolle der grünen Bundestagsfraktion 1983 bis 1987 herausgebracht, ergänzt um eine Fülle von Dokumenten, die Licht auf das Innere grüner Prozesse werfen. Man sollte die Chance nutzen, um zu verstehen, dass es für die Grünen gut war, nicht bei ihren Kinderkrankheiten stehen zu bleiben. 
Es gab bei den Grünen von Anfang an Pioniere der Spurensicherung, wie Robert Camp, die sammelten und sichteten, während in der Partei der Kampf um Macht, Richtung, ja das Überleben tobte. Inzwischen ist auch das Archiv ausgedehnt, professionalisiert, etabliert und bewahrt die Bestände ihrer Großen, gemessen in Metern. In enger Zusammenarbeit mit dem heutigen Archiv-Leiter Christoph Becker-Schaum und seinen Mitarbeitern entstand nun eine besonders wertvolle Dokumentation - schließlich war die Bundestagsfraktion seit ihrem Bestehen, zusammen mit den Parteitagen, zentrales Organ fortschreitender Richtungsbestimmung, und - mehr als die anderen Gremien - Vorreiter eines Professionalisierungsprozesses der Bundespartei.

Nicht zuletzt weil die Fraktionssitzungen in den 1980er Jahren weitgehend öffentlich waren, kann es nicht verwundern, dass es durch die Protokolle keine Überraschungen im Großen gibt. Die Geschichte der Grünen muss nicht neu, aber sie kann nun anders geschrieben werden: genauer, farbiger, noch näher an den Akteuren. Gerade in Kenntnis dessen, was sich gegen anhaltenden Widerstand in den 1990er Jahren als Reformpolitik durchgesetzt hat, erhalten viele hier zugänglich gemachte Fakten Relevanz und Stellenwert. Am Beispiel: Nur zwei Abgeordnete hätten am 8. März 1983 im Fraktionsplenum ihre Stimme anders abgeben müssen, dann hätte die Fraktion eine Spitze mit einem Sprecher und zwei Stellvertretern bekommen. Tatsächlich startete man mit drei gleichberechtigten Sprechern - eine Einladung, die Strömungskämpfe öffentlich wirksam gerade auch auf dieser Ebene auszutragen, und ein Erschwernis, persönlich Verantwortung zu übernehmen (nach innen und außen). Weniger wichtig, aber kulturell interessant ist die Aufforderung in der Fraktionssitzung vom 15. März 1983, den Fahrdienst des Bundestags nicht nachts nach Kneipenbesuch zu beanspruchen. Und so geht es weiter: eine Fundgrube aufschlussreicher Details aus dem ungewöhnlichen Experiment, soziale Bewegungen mit dem etablierten Parlamentarismus zusammenzubringen.

Das Brot der frühen Jahre bestand auch aus regelrechter Feindschaft, Hysterie und Kampf bis aufs Messer. Viele hatten schon vorher radikale Positionen bezogen, nicht wenige der wichtigen Akteure in den Sonderwelten der K-Gruppen. Von den Richtungskämpfen, die die Partei auch hätten scheitern lassen können, blieb die Bundestagsfraktion nicht verschont. Die hier dokumentierten Fraktionsvollversammlungen luden zum Ideologienstreit ein. Die sachlich-souveräne Einleitung von Helge Heidemeyer rückt eher die positiven Seiten der Entwicklung in den Vordergrund: die Fortschritte bei der fortlaufenden Professionalisierung, die Beiträge zu inhaltlicher Profilschärfung bei Umwelt-, Atom-, Rüstungspolitik, bei der Gentechnik und Frauenfragen, nicht zuletzt beim hochambitionierten „Umbauprogramm“, die, wie es heißt, „Erfolge auf den zweiten Blick“ (S. XXVI ff.). Die „imponierende Leistungsbilanz“ (S. XXXIV) der Bundestagsfraktion ist unbestritten, sie beantwortet aber noch nicht die Frage nach Zahl und Reichweite der Innovationen. Wer hat sich mehr verändert: die bestehenden Strukturen oder die Grünen selbst? Zusammen mit den Dokumenten liefern Einleitung und Kommentierung nun die Grundlage für die noch ausstehende Monografie zur grünen Bundestagsfraktion mit der umfassenden und abgewogenen Würdigung ihrer Arbeit seit 1983.

Anfang der 80er Jahre befand man sich im Übergang zum Vierparteiensystem, was heute, im Zeichen des Fünfparteiensystems, zusätzliches Interesse findet. Die Grünen haben gegen den etablierten Dreiparteien-Club der 60er und 70er Jahre erfolgreich Kämpfe durchgestanden, von deren Ergebnis nachfolgende Kleinparteien profitieren können. Auch beim Agenda-Setting waren sie erfolgreich (Umwelt-, Atom-, Friedens-, Frauenpolitik etc.). 
Neu waren die Personen (aus den Bewegungen kommend) und die Themen, aber sie schufen kein neues Parlament. Ihnen gelang es, die Oppositionsarbeit zu beleben (mehr Große und Kleine Anfragen, mehr Aktuelle Stunden) und damit Kritik, Kontrolle und Alternative als Oppositionsfunktionen sichtbarer zu machen. Aber das sagt nur, dass die Grünen bienenfleißig waren, nicht, dass sie Strukturen verändert hätten. Eckart Stratmann, damals einer der führenden Fraktionslinken, brachte es auf den Punkt: „Im Grunde kommt man durch Erfahrung zu Strukturen, die die anderen schon lange haben - und die wir lange bekämpft haben."

Mit dem Buch wird die parlamentarische Frühgeschichte der Grünen exzellent erschlossen. Zustande gekommen ist eine überaus sorgfältige, zuverlässige Edition, die sich phantasievoll, aber präzise auf die fragmentierten Realitäten alternativer Bürokratie, wie sie die frühen Fraktionsjahre prägten, einlässt. Dazu gehören: eine behutsame, sachverständige Kommentierung; ein Glossar, das hilft, sich rasch in dem Gewirr von Untergruppierungen zurecht zu finden, das die grüne von den etablierten Fraktionen unterschied; ein Personenregister und vollständige Kurzbiographien von Abgeordneten; transparente Kriterien der Dokumentenauswahl.

Für die Verfeinerung unseres historischen Wissens über die Grünen liegt damit eine hervorragende Quelle vor. Sie kann und sollte auch weitere zeithistorische Forschung anregen. Es ist wünschenswert, dass diese Edition über 1987 hinaus fortgeführt wird.

Joachim Raschke

\section{Neue Wege der Parteienfinanzierung}

Morlok, Martin, Ulrich von Alemann und Thilo Streit (Hrsg.): Sponsoring - ein neuer Königsweg der Parteienfinanzierung?, Beiträge des Symposions des Instituts für Deutsches und Europäisches Parteienrecht und Parteienforschung (PRuF) am 15./16. Oktober 2004 (Schriften zum Parteienrecht und zur Parteienforschung, Band 32), Nomos Verlagsgesellschaft, Baden-Baden 2007, 154 Seiten, € 29,-.

Reffken, Hendrik: Politische Parteien und ihre Beteiligungen an Medienunternehmen. Eine Untersuchung aus verfassungsrechtlicher Sicht (Studien und Materialien zur Verfassungsgerichtsbarkeit, Band 103), Nomos Verlagsgesellschaft, Baden-Baden 2007, 455 Seiten, € 86,-.

Schindler, Alexandra: Die Partei als Unternehmer (Schriften zum Parteienrecht und zur Parteienforschung, Band 31), Nomos Verlagsgesellschaft, Baden-Baden 2007, 228 Seiten, € 49,-.

Volksparteien zählen zweifelsohne zu den wichtigsten Errungenschaften demokratischer Massengesellschaften. Sie unterscheiden sich von anderen Parteitypen durch ihre Integrationsfähigkeit. Anders als so genannte Klientelparteien richten sich Volksparteien nicht an bestimmte Klassen oder Schichten der Bevölkerung, sondern an jedermann, und zwar sowohl hinsichtlich der Mitgliedschaft als auch der Wählbarkeit. Sie leben von der Zustimmung aus den unterschiedlichen Milieus und nähren sich von der Chance, durch ihr programmatisches und personelles Angebot alte Anhänger zu mobilisieren und neue hinzuzugewinnen - über alle Grenzen sozioökonomischer Unterschiede, religiöser Prägun- 